rialismus« (185)? Die Antwort fällt ambivalent aus. Selbstverständlich sind die Entdeckungsunternehmen mit ihrem erheblichen finanziellen und logistischen Aufwand Teil der globalen europäischen Expansion. Indem sie Landkarten erstellen oder Handelswege und Posten etablieren helfen, leisten die indigenen Begleiter einen wesentlichen Beitrag dazu. Ihre Motive sind aber biographischen und lokalen Begrenzungen unterworfen. Wie man schwerlich Alexander von Humboldts Südamerika-Reise, die ihren Erfolg auch den imperialistischen Inter- essen Spaniens verdankt, insgesamt als imperialistisches Unternehmen wird abtun können, so wird man auch die manchmal aus der Not geborenen, manchmal kalkulierten Entscheidungen Indigener, sich an den Expeditionen zu beteiligen, nicht allein im Licht ihres Nutzens für den europäischen Imperialismus sehen können. Im Gegenteil, die Erforschung ihrer Leistungen ist eine Aufgabe, die auch zur Würdigung ihrer komplizierten Geschichte beiträgt.

Hans-Christian Riechers

\title{
Esaïe Djomo (Hg.): Lieder der alten Afrikaner. Anthologie deutschsprachiger Koloniallyrik 1884-1945. Mit einem Vorwort v. Esaïe Djomo
}

St. Ingbert: Röhrig 2017 - ISBN 978-3-86110-631-9 - 29,00€

Im Jahr 1992 ist bei Röhrig als 31. Band der Saarbrücker Beiträge zur Literaturwissenschaft die Dissertation Esaïe Djomos mit dem Titel »Des Deutschen Feld, es ist die Welt!« Pangermanismus in der Literatur des Kaiserreichs, dargestellt am Beispiel der deutschen Koloniallyrik. Ein Beitrag zur Literatur im historischen Kontext erschienen. Ein Vierteljahrhundert später legt der Verfasser nun im selben Verlag eine Anthologie vor, deren Umfang im Wesentlichen dem damals untersuchten Textkorpus entspricht, wie das Vorwort der Lieder der alten Afrikaner eingangs auch sogleich betont (vgl. 17). Insofern scheint es durchaus angemessen, zunächst einige Worte über die Dissertation von $1992 \mathrm{zu}$ verlieren, zumal deren Konzeption die Auswahl der Texte auch für die Anthologie von 2017 offensichtlich entscheidend bestimmt.
Wie bereits der Untertitel der älteren Untersuchung hervorhebt, besteht deren Bedeutung in erster Linie in der Einbettung des kolonialen Diskurses in die Gedankenwelt des Pangermanismus, der vom späten Kaiserreich bis zum Beginn des Dritten Reiches stetig an Einfluss gewinnt. Dazu weist Djomo in den untersuchten Texten eine Typologie der Themen nach, die den pangermanistischen Kolonialdiskurs in besonderem Maße kennzeichnen, wie die Lebensraumfrage, die Kriegsbegeisterung, die Flottenpolitik oder die Germanisierung der Kolonien. Als dominantes stilistisches Mittel der untersuchten Koloniallyrik gilt Djomo die Kontrafaktur, die sich insbesondere in der Form äußert, dass zahlreiche Gedichte nach Strophenform und Melodie bekannter patriotischer Gesänge oder populärer Volkslieder verfasst werden. Neben 
der überzeugenden Analyse der Koloniallyrik als Ausdruck pangermanistischer Vorstellungen besteht das Verdienst der Studie in erster Linie darin, dass sie der Literaturwissenschaft Texte zugänglich macht, die zu einem großen Teil weit verstreut in den Periodika der kolonialen Verbände und Gesellschaften wie der Kolonialen Zeitschrift, Kolonie und Heimat in Wort und Bild oder der Brücke zur Heimat erschienen sind.

Um der Literaturwissenschaft nach der thematischen Anregung durch die Studie von 1992 nun eine »ertragreiche wissenschaftliche Nutzung « (17) des zusammengestellten Textkorpus zu ermöglichen, wird dieses in der vorliegenden Anthologie präsentiert. Diese legt den Schwerpunkt auf die »in Zeitschriften veröffentlichen Gedichte und Lieder« (ebd.) und verweist für die in zeitgenössischen Gedichtsammlungen erschienene koloniale Lyrik auf deren Wiederabdruck in Arne Schöferts Deutsche Koloniallyrik. Lieder und Gedichte (vgl. 2012), die Djomo ausdrücklich als »lobenswertes Unternehmen« bezeichnet (17). Warum Djomo sich vor diesem Hintergrund nicht allein auf die Zeitschriftenveröffentlichungen konzentriert oder sich zumindest auf diejenigen Sammlungen beschränkt, die nicht bereits durch Schöfert wieder zugänglich gemacht worden sind, wird leider an keiner Stelle begründet - vielleicht rechtfertigt jedoch die geringe Verbreitung der Deutschen Koloniallyrik in den einschlägigen wissenschaftlichen Bibliotheken Djomos Vorgehen.

Vor dem Hintergrund der Konzentration auf die Zeitschriften kolonialer Verbände sowie die zeitgenössischen Anthologien zur Koloniallyrik, deren
Herausgebern dasselbe Interesse am kolonialen Diskurs unterstellt werden darf, ist es nun freilich wenig verwunderlich, wenn Djomo durchweg eine affirmative Haltung zum Kolonialismus konstatieren muss, wie sowohl das Vorwort als auch der Klappentext feststellen:

Die überwältigende Mehrheit der Texte sind [sic] nach wie vor eindeutige affirmative Stellungnahmen zur Kolonisation. Umso mehr wird der Leser sich freuen, die drei interessanten antikolonialen Gedichte von Karl Schnog, Hermann Landois und das anonyme Gedicht Bibel und Flinte, das bereits 1898 erschienen war, in diesem Band zu entdecken. (17)

Bibel und Flinte (vgl. 28) stammt nun eben gerade nicht aus einer der sonst herangezogenen Zeitschriften oder Anthologien, sondern aus dem nur für dieses eine Gedicht konsultierten Demokratischen Liederbuch (vgl. O.A. 1898), das Kongo-Lied von Hermann Landois (vgl. 215f.) sowie Karl Schnogs Wir brauchen Kolonien (vgl. 340) werden gar nach der dritten Auflage einer von Horst Gründer erstmals im Jahr 1999 herausgegebenen Anthologie zitiert (vgl. Gründer 2006). Ob hier nicht die Voraussetzungen und Grundlagen der Recherche ein reichlich verzerrtes Ergebnis quasi vorwegnehmen, müsste zumindest diskutiert werden.

Weit problematischer als die Auswahl der Texte ist jedoch ihre Präsentation in den Liedern der alten Afrikaner, die ja ausdrücklich auf die »wissenschaftliche Nutzung « hin ausgerichtet sind, weshalb der Herausgeber »jedes Gedicht mit Quellenangaben gleich nach dem Text versehen« habe (17) 
Diese Quellenangaben erscheinen als Siglen, die in einem angehängten Quellenverzeichnis aufgeschlüsselt werden, wo aber beispielsweise das bereits erwähnte Demokratische Liederbuch als »DL« abgekürzt wird (365), während sich unter dem Text selbst die Sigle »DLB « findet (28). Dass hier eine Seitenzahl fehlt, ist keine Ausnahme, sondern im Fall der mit den Siglen A1 bis A14 abgekürzten Anthologien und Werkausgaben (bei denen es erstaunlicherweise kein $\mathrm{A}_{3}$, dafür aber ein außerhalb jeglicher Systematik stehendes B14 gibt) leider die Regel; zuweilen finden sich neben oder anstelle der angeführten Siglen kryptische Abkürzungen wie »Ab« (32), »Ad« (51) oder $» B c \ll$ (328). Für die Zeitschriften wird neben der Seitenzahl lediglich die Bandnummer angegeben, was den Benutzer zwingt, zur Eruierung des ja für die Einordnung doch entscheidenden Erscheinungsjahres stets das Quellenverzeichnis zu konsultieren. Djomo scheint selbst bemerkt zu haben, wie wenig benutzerfreundlich diese Vorgehensweise ist, zumindest finden sich in der zweiten (kürzeren) Abteilung zu Weimarer Republik und Drittem Reich anders als in der ersten zum Kaiserreich die Jahresangaben zumindest bei den Nachweisen, die Zeitschriften betreffen.

Dagegen verzichtet der Herausgeber beim Abdruck der Gedichte auf jegliche Kommentierung derselben; einzelne als Fußnoten erscheinende Bemerkungen stehen offenbar bereits in der Quelle, aus der die Anthologie jeweils schöpft (vgl. z.B. 27, 60, 72, 172f. oder 188) - und in einem Fall übernimmt Djomo lediglich die äußerst kreativen Fußnotenzeichen des Originals im Haupttext (von »+)《über
$» \iint \mathbb{\int} \ll$ und $» *+\ll$ bis $\left.»+\mathbb{\int}\right) \ll$ ), druckt aber die dazugehörigen Fußnoten selbst nicht ab (113-143). Insbesondere von den Verfassern der Gedichte erfährt der Leser häufig nicht einmal den vollständigen Namen; zur Verfasserschaft der unter einem Pseudonym erschienenen Gedichte äußert der Herausgeber sich ebenfalls nicht. Rätselhaft bleibt auch die Reihenfolge, in der die einzelnen Gedichte abgedruckt werden: Diese scheint zunächst vage einer alphabetischen Auflistung nach den Verfassernamen $\mathrm{zu}$ folgen, was das angehängte Namensregister zu einer bloßen Wiederholung des Inhaltsverzeichnisses macht; allerdings wird die alphabetische Anordnung ohne erkennbares System häufig unter- und durchbrochen. Selbst Gedichte ein und desselben Verfassers erscheinen nicht immer unmittelbar nacheinander (vgl. beispielsweise 160 u. 217) und wenn dies doch der Fall ist, wird der Autor mal nur vor dem ersten Gedicht genannt, mal wird der Name zusätzlich vor dem vierten, siebten oder zehnten Gedicht wiederholt.

Schlimmer als all diese ärgerlichen Ungenauigkeiten im Aufbau der Anthologie und auch als das unschöne Layout, das für die Autorennamen munter zwischen Kapitälchen und normalem Druck wechselt, die letzten Worte überlanger Verse mal rechts- und mal linksbündig unter den Vers setzt (so 184 u. 320, besser 208 u. 319), ein einzelnes Gedicht in einer anderen Schriftgröße präsentiert als alle anderen (vgl. 311), einzelne Buchstaben gar nicht aufs Papier bringt (58: anstelle von »R uber« lies »Räuber«; 81: anstelle von »Aeug ein« lies »Aeuglein«), unmotivierten Fettdruck verwendet (244: »wehrlo- 
sen«) oder für einige Seiten (vgl. [316], [317], [318]) gar auf eine Paginierung verzichtet, ist freilich die immense Anzahl an offensichtlichen Druckfehlern, die der Text aufweist und die jeder Lektor auch ohne den zugegebenermaßen aufwändigen Vergleich mit der jeweiligen Quelle hätte finden können. Für den (unwahrscheinlichen) Fall, dass sich diese Fehler sämtlich bereits in der Quelle finden sollten, wäre vom Editor zwingend entweder die Herstellung eines lesbaren Textes mit Apparat oder zumindest eine Markierung der offensichtlichen Fehler durch den Vermerk »sic« o.Ä. zu leisten gewesen. Um einen Eindruck vom Ausmaß dieser Problematik zu vermitteln, soll im Folgenden eine Liste der offensichtlichsten Korrigenda präsentiert werden:

Anstelle von »Sank« lies »Sang« (37), anstelle von »wir« lies »wird « (45), anstelle von »Reichen« lies »Reiche « (50), anstelle von »Verdunke'n« lies »Verdunkel'n« (58), anstelle von »mann« lies »Mann«, anstelle von »di« lies »dir« (beide 81, der erste Fehler genauso auch noch einmal 226), anstelle von »find « lies »sind « (91), anstelle von »tag « lies »Tag« (99), anstelle von »einh« lies »ein« (102), anstelle von »erlagner« lies »erlogner« (104), anstelle von »den« lies »denn« (111), anstelle von »Bruder« lies »Brüder« (126), anstelle von »Schaun« lies »schaun« (133), anstelle von »fremden Kund« lies »Fremden kund«, anstelle von »Stumm« lies »stumm« (beide 134) ... Bedauerlicherweise ergäbe sich beim Blick auf die restlichen Seiten der Anthologie ein ähnliches Bild.

Man könnte diesen Befund nun zum Anlass nehmen, wortreich die allfälligen Sparmaßnahmen der Ver- lage und insbesondere das langsame Aussterben eines kompetenten Lektorats zu beklagen, bliebe einem solchen laudator temporis acti nicht das Wort im Halse stecken, sobald er bemerkt, dass sich beispielsweise der »Winkingergeist « bereits in Djomos Dissertation findet und im Jahr 1992 bei Röhrig also auch nicht wesentlich gründlicher gearbeitet worden ist. Dennoch stellen zahlreiche der hier aufgelisteten Fehler nicht einfach nur lässliche Versehen dar - sofern es so etwas bei wissenschaftlichen Editionen überhaupt gibt -; vielmehr bedingen sie mitunter gravierende Sinnentstellungen, wie der folgende Lapsus illustrieren kann (der ebenfalls bereits 1992 aufgetreten ist). Die erste Strophe von Hans Heinrich Lemméls »Der Jugend die Zukunft« lautet in den Liedern der alten Afrikaner:

Ich kenne fern manch weites, weites Land,

Das deutscher Fleiß zu erstem Blühen rief;

Wo deutscher Flug [sic] geführt von deutscher Hand,

Den Boden brach, der lange fruchtlos schlief;

Schwer war der Weg, doch rastlos varwärts [sic] trug

Die Kolonien deutschen Geistes Flug.

Dass anstelle von »varwärts « natürlich »vorwärts« zu lesen ist, mag noch jedem Benutzer der Anthologie unmittelbar einleuchten, obgleich selbst dieser offensichtliche Lesefehler das wissenschaftliche Zitieren nach Djomos Edition erheblich erschwert; die Variante »Flug « anstelle von »Pflug« ist jedoch auch insofern gefährlich, als sie über die Alliteration zu »Fleiß« im vo- 
rangegangenen Vers zunächst einmal plausibel erscheint und als lectio difficilior eine gewisse Attraktivität ausstrahlt, die den ein oder anderen Leser durchaus verführen könnte, ihr auf den vielzitierten Holzweg zu folgen. Groteske Züge nimmt die Editionspraxis in den Liedern der alten Afrikaner jedoch dann an, wenn das vorliegende Gedicht mit identischer Autoren- und Quellenangabe einige Seiten später erneut abgedruckt wird - und zwar mit den (korrekten) Lesarten »Pflug « und »vorwärts« $\left(35^{6}\right)$.

Abschließend ist also ein durchaus gemischtes Fazit zu ziehen: Djomos Textauswahl überzeugt vor dem Hintergrund der Fragestellung, mit der er sich 1992 dem Thema genähert hat; vielleicht hätten ein Verweis auf die Schnittmengen von kolonialem und pangermanistischem Diskurs im Titel auch der Anthologie von 2017 und dazu ein etwas ausführlicheres Vor- wort die Stärken dieser Textsammlung noch deutlicher hervorheben können. So verschafft eine Lektüre der Texte einen guten Überblick über die Verflechtung der beiden genannten Diskurse, bleibt aber aufgrund der fehlenden Kommentierung, zu der man die Ergebnisse der Studie von 1992 durchaus hätte umarbeiten können, zumindest für den nicht tiefer in das Thema eingearbeiteten Leser eine mühsame Aufgabe. Besonders bedauerlich aber ist der Umstand, dass Djomos Anthologie aufgrund ihrer zahlreichen Fehler und ihrer letztlich wissenschaftlichen Standards nicht entsprechenden Editionsprinzipien dem interessierten Wissenschaftler den Gang zu den Quellen dann eben doch nicht ersparen kann - und damit letztlich ihren Sinn und Zweck in einem wesentlichen Punkt verfehlt.

\section{Heiko Ullrich}

\title{
Literatur
}

Gründer, Horst (Hg.; ${ }^{32006)}$ : »da und dort ein junges Deutschland gründen«. Rassismus, Kolonien und kolonialer Gedanke vom 16. bis zum 20. Jahrhundert. München. Schöfert, Arne (22012): Deutsche Koloniallyrik. Lieder und Gedichte. Saarbrücken.

O. A. (1898): Demokratisches Liederbuch. Stuttgart.

\section{Constanze Weth / Kasper Juffermans (Hg.): The Tyranny of Writing. Ideologies of the written word}

\author{
London: Bloomsbury - ISBN 978-1-474-29244-3 - 102,60£
}

Writing has been largely ignored, and even denied, as an object of study in sociolinguistics (see for instance discussions in Sebba/Mahootian/Jonsson 2012; Lillis/McKinney 2013). The Tyranny of Writing. Ideologies of the written word is therefore a welcome input to the recent sociolinguistic investigation of written language, ironically de- parting from Saussure's metaphorical argument against writing as an object of linguistic research, la tyrannie de la lettre. In this edited volume the metaphor is however used in the sense put forward by Florian Coulmas: »the >tyranny< of writing is a social reality that ought to be studied as such « (2013: 8). The editors Constanze Weth 